\title{
FUNCIÓN DE PRODUCCIÓN DE CEMENTO: CASO UNIÓN CEMENTERA NACIONAL - PLANTA CHIMBORAZO
}

\author{
FUNCTION OF CEMENT PRODUCTION: CASE UNION CEMENTERA NACIONAL - PLANT CHIMBORAZO
}

Karina Alexandra Álvarez Basantes ${ }^{1}$

Gerardo Mauricio Zurita Vaca ${ }^{2}$

Doris Nataly Gallegos Santillán 3

\section{Resumen}

Desde una función de producción Cobb Douglas, se determina cómo contribuye cada uno de los factores productivos (capital y trabajo) en la productividad de la planta Chimborazo de la Unión Cementera Nacional. Se parte de la hipótesis que la combinación de los dos factores interviene ampliamente en la producción de cemento. Se demuestra, además, que la empresa requiere aprovechar en mayor medida su capacidad instalada y alcanzar sus niveles óptimos de producción.

\section{Palabras clave}

Empresa, factores productivos, función de producción.

\section{Abstract}

From a Cobb Douglas production function, we determine how each of the productive factors (capital and labor) contributes to the productivity of the Chimborazo plant of the National Cement Union. It is assumed that the combination of the two factors intervenes widely in the production of cement. It shows that it is necessary for the company to take better advantage of its installed capacity and reach its optimum levels of cement production.

\section{Keywords}

Company, production factors, production function.

\footnotetext{
${ }^{1}$ Investigadora independiente (Ecuador). Correo electrónico: kriny_10@hormail.com

2 Universidad Nacional de Chimborazo (Ecuador). Correo electrónico: gzurita@unach.edu.ec

${ }^{3}$ Universidad Nacional de Chimborazo (Ecuador). Correo electrónico: dgallegos@unach.edu.ec
} 


\section{Introducción}

La función de producción Cobb Douglas ha sido aplicada a nivel macroeconómico, a fin de estimar la producción de la economía costarricense (Azofeifa y Villanueva, 1996), o de la economía española (Bellod, 2011). También se ha utilizado para determinar el cambio técnico y la eficiencia técnica en Latinoamérica (Méndez et al., 2013), o para evaluar el PIB potencial y la productividad total de los factores en México (Acevedo, 2008). Desde el punto microeconómico, se ha empleado para estimar la producción de algodón del Valle de Juárez (Cortázar y Montaño, 2011), o para el teatro en Colombia (Parra, 2011). A nivel de firmas peruanas, se aplicó para determinar su productividad sectorial (Céspedes et al., 2014), o conocer la competencia intra-industria, aglomeraciones regionales y crecimiento económico en el caso de la industria de alimentos en Colombia (Camacho y Ríos, 2010).

Como señala Aguado (2011), una función de producción expresa la relación existente entre las diferentes combinaciones de factores productivos y la cantidad máxima del bien que puede obtenerse con ellas. Esta función se la puede estudiar a corto y a largo plazo. En el corto plazo, uno de los factores, generalmente el capital, permanece constante, por lo que la función de producción expresará la cantidad de producto que podemos obtener en función al incremento o disminución del factor trabajo para una tecnología dada, mientras que en el largo plazo todos los factores están sujetos a variaciones.

Al respecto Dominick (2009), considera a la función de producción como una ecuación, tabla o gráfica que indica la cantidad máxima que puede producirse por unidad de tiempo, considerando un conjunto de insumos, cuando se utilizan las mejores técnicas de producción disponibles. La relación entre los factores de producción y los bienes con una función de producción se expresaría de la siguiente forma:

$q=f(k, l, m, \ldots)$

Donde " $q$ representa la producción de un determinado bien durante un periodo, $k$ representa la maquinaria o capital utilizada durante el periodo, $l$ representa las horas de trabajo, $m$ representa las materias primas empleadas, y la notación indica la posibilidad de que otras variables afecten el proceso de producción" (Nicholson, 2008, p.183). En este sentido, la función de producción es la representación de la combinación de factores o insumos productivos, con la finalidad de obtener un determinado volumen de producción, ya sea de un bien o servicio. En virtud que en el corto plazo se considera el nivel de tecnología dada y el capital constante, se procede a analizar los cambios que genera en la producción el incremento o disminución de personal. Estas variaciones del factor trabajo se analiza a través del producto marginal y del producto medio.

En este marco, el presente trabajo estudia desde una perspectiva microeconómica la función de producción de la planta Chimborazo de la Unión Cementera Nacional CEM, a fin de estimar las cantidades óptimas de los factores productivos que requiere un determinado volumen de producción de cemento, a partir de una función Cobb Douglas, en la cual el volumen de toneladas métricas de cemento producidas están en función del capital o capacidad instalada (edificios, maquinarias, equipos y herramientas que interviene en la producción de cemento) y los trabajadores, tanto operativos como administrativos, quienes desde sus puestos de trabajo contribuyen a la productividad de la empresa.

La pregunta es relevante, si tenemos en cuenta los procesos de concentración que ha presentado la industria cementera en el Ecuador, durante las últimas décadas. En el sector privado, La compañía Holcim se fusionó con Lafarge. En el sector público, Unión Cementera Nacional -CEM- se originó de la fusión entre Cemento Chimborazo C.A. e Industrias Guapan S.A. Para incrementar su producción, cada una de ellas ha invertido importantes capitales esperando mejorar su capacidad instalada en respuesta a la demanda insatisfecha del producto en el mercado. Sin embargo, al expandirse, estas empresas no han conseguido una optimización eficiente de sus recursos, tanto desde el punto de vista técnico como administrativo, subutilizando su nueva capacidad instalada.

\section{Método}

La estimación de la función de producción de UCEM CEM, se planteó considerando que la función de producción Cobb-Douglas relaciona cantidades de producto con cantidades de factores utilizados de la siguiente manera:

$y_{i}=\mathrm{b}_{1} x_{2 i}^{\mathrm{b}_{2}} x_{3 i}^{\mathrm{b}_{3}} e^{u_{i}}$

Donde: $y$ es el volumen de producción, X2 el factor 
trabajo, X3 el factor capital, $u$ el término de perturbación estocástica, y e la base del logaritmo natural.

Se parte del supuesto que la empresa minimiza sus costos para producir " $y$ " toneladas métricas de cemento, lo cual implica que existe una función de costos que relaciona el costo mínimo posible de producir la cantidad óptima de cemento, con los precios de los factores utilizados y el estado de conocimiento tecnológico. Esta función se representa de la siguiente manera:

$C=p_{2} x_{2}+p_{3} x_{3}$

Donde: $P_{2}$ es precio del capital, y $P_{3}$ es el precio del trabajo.

Esto permite obtener las funciones de demanda óptima de los factores, y al sustituirlas en la función de costos se llega a:

$C=k^{*} y^{\frac{1}{r}} p_{2}^{\frac{\mathrm{a} 2}{\mathrm{~g}}} p_{3}^{\frac{\mathrm{a} 3}{\mathrm{~g}}}$

Para estimar la función, se procede a su transformación logarítmica con la finalidad de hacerla lineal. Posteriormente se aplicaron mínimos cuadrados ordinarios para establecer el comportamiento lineal de las variables capital y trabajo, sobre la siguiente especificación:

$\ln y_{i}=\ln \mathrm{b}_{1}+\mathrm{b}_{2} \ln X 2_{i}+\mathrm{b}_{3} \ln X 3_{i}+u_{i}=\mathrm{b}_{0}+\mathrm{b}_{2} \ln X 2_{i}+\mathrm{b}_{3} \ln X 3_{i}$

Donde: $\beta 0=\ln \beta 1$.

\section{Resultados}

La planta Chimborazo de la Unión Cementera Nacional UCEM CEM produce y comercializa el Cemento Portland Puzolánico IP en sacos de $50 \mathrm{~kg}$. y Cemento Portland Tipo II que se distribuye a granel; también comercializa productos intermedios como la Puzolana. En 2011 comenzó la construcción y puesta en marcha de la nueva línea de molienda y despacho de cemento de la planta Chimborazo, para lo cual invirtió aproximadamente 40 millones de dólares. Esta modernización permitió el incremento significativo del rendimiento, así como también de la capacidad de abastecimiento al mercado local y nacional, aumentando su capacidad de producción de 330 mil a un millón de toneladas de cemento por año.
El volumen de producción de la planta Chimborazo de la Unión Cementera Nacional UCEM CEM pasó de 327.747,02 TM registrado en el año 2010, a 545.141,95 TM en el 2014, lo cual en términos porcentuales significa un incremento de $66,33 \%$, fruto de la nueva capacidad instalada, como por ejemplo el molino tubular con una capacidad máxima de hasta 100 toneladas de cemento por hora, un silo de almacenamiento para despacho a granel con cinco compartimientos, el cual es utilizado para almacenar los distintos tipos de cemento, el mismo que tiene una altura de 63 metros.

Figura 1. Volumen de cemento producido (toneladas métricas). Periodo 2010-2014.

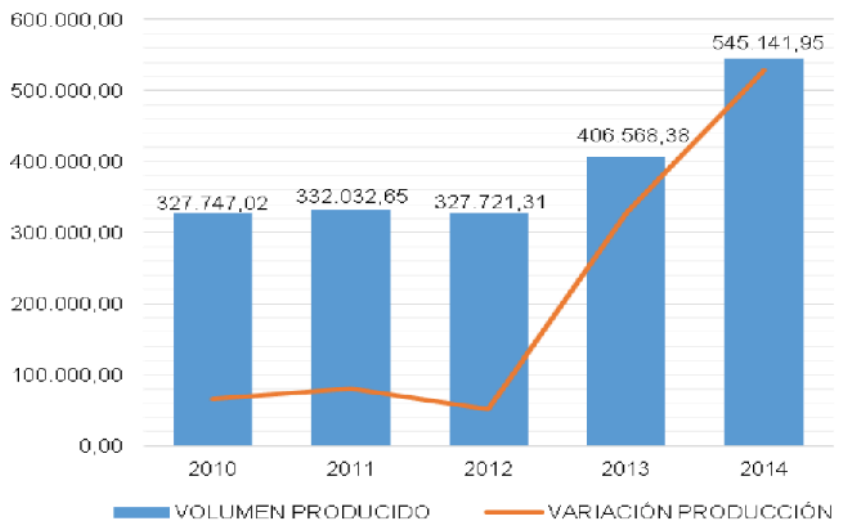

Fuente: elaboración propia sobre reporte de producción y consumo de materias primas mensuales 2010-2014.

La figura que antecede muestra el comportamiento de la producción durante el periodo 2010-2014, en el que se puede observar un crecimiento significativo a partir del año 2012. El crecimiento registrado en el año 2013 fue de 78.847,07 TM más que en el 2012, es decir un incremento del 24,06\%; mientras que, entre los años 2013-2014 la producción de cemento incrementó en 138.573,57 TM, lo cual representó un crecimiento del $34,08 \%$.

Los incrementos de producción de la planta Chimborazo de la Unión Cementera Nacional -UCEM CEM-, permitieron que esta pudiera expandirse y atender la demanda del mercado de la construcción y, paralelamente, ser más competitivos respecto de las demás empresas del sector cementero a nivel local, nacional e incluso internacional. Los volúmenes de producción alcanzados en los distintos periodos analizados fueron posibles gracias al trabajo conjunto del personal, tanto administrativo como operativo, que labora en la planta Chimborazo de la Unión Cementera Nacional UCEM CEM. 
Figura 2. Promedio mensual de trabajadores (número de personas). Periodo 2010-2014.

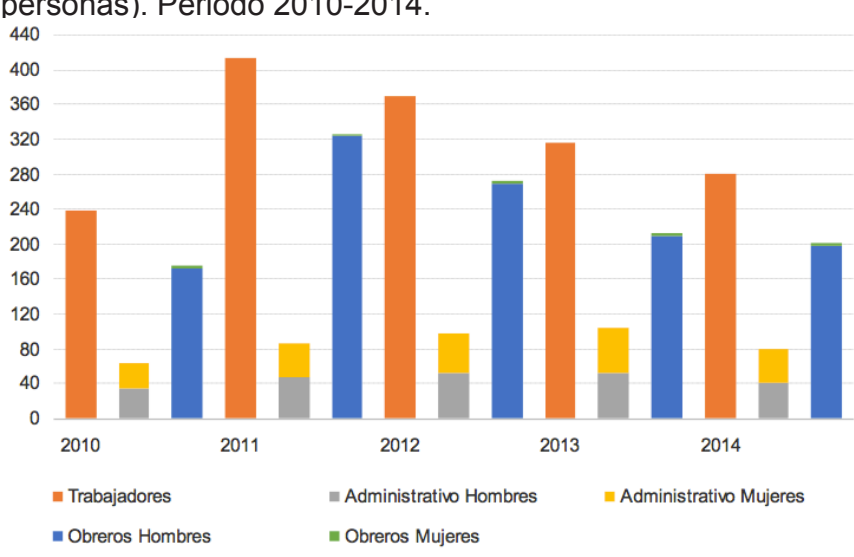

Fuente: elaboración propia sobre indicadores de nómina mensuales 2010-2014 - Gerencia de Talento Humano \& DO.

Según el reporte de indicadores mensuales de nómina, se ha determinado que el promedio mensual de trabajadores en el año 2010 era de 238 personas, de las cuales en actividades administrativas constan 34 hombres y 30 mujeres, mientras que en actividades operativas encontramos 171 hombres y 3 mujeres. En el año 2011, el promedio mensual de trabajadores correspondía a 413 personas, de las cuales en actividades administrativas constan 47 hombres y 39 mujeres, mientras que a las actividades operativas se dedican 324 hombres y 3 mujeres.

Durante el año 2012, el promedio mensual de trabajadores es de 369 personas, de ellas, en actividades administrativas se encuentran 51 hombres y 46 mujeres, mientras que se dedican a actividades operativas 270 hombres y 2 mujeres. Cabe mencionar que el incremento de personal registrado en los años 2011 y 2012 fue debido a que durante estos periodos estuvo operativa el área de prefabricados, que se dedicaba a la producción de durmientes para la remodelación del ferrocarril.

En el año 2013, en la planta industrial trabajaban 316 personas, de las cuales en actividades administrativas se encontraban 53 hombres y 51 mujeres, mientras que en actividades operativas estaban 210 hombres y 3 mujeres. Finalmente en el año 2014, el personal mensual promedio es de 281 personas, de la cuales en actividades administrativas se encuentran 40 son hombres y 39 son mujeres mientras que se dedican a actividades operativas 198 hombres y 3 mujeres.
Tabla 1. Horas hombre trabajadas (horas anuales acumuladas) periodo $2010-2014$.

\begin{tabular}{|c|c|c|c|c|c|c|c|c|}
\hline \multirow{2}{*}{ AÑO } & \multirow{2}{*}{$\begin{array}{l}\text { HORAS DE } \\
\text { TRABAJO }\end{array}$} & \multicolumn{2}{|c|}{ ADMINISTRATIVO } & \multirow{2}{*}{$\begin{array}{l}\text { TOTAL } \\
\text { ADM. }\end{array}$} & \multicolumn{2}{|c|}{ OBREROS } & \multirow{2}{*}{$\begin{array}{l}\text { TOTAL } \\
\text { OBR. }\end{array}$} & \multirow{2}{*}{ TOTAL } \\
\hline & & HOMBRES & MUJERES & & HOMBRES & MUJERES & & \\
\hline \multirow{2}{*}{2010} & NORMAL & 66.166 & 57.240 & \multirow{2}{*}{139.887} & 331.810 & 6.444 & \multirow{2}{*}{449.715} & \multirow{2}{*}{589.602} \\
\hline & EXTRAS & 6.459 & 10.022 & & 110.732 & 729 & & \\
\hline \multirow{2}{*}{2011} & NORMAL & 88.229 & 73.750 & \multirow{2}{*}{182.729} & 637.211 & 6.178 & \multirow{2}{*}{836.275} & \multirow{2}{*}{1.019 .004} \\
\hline & EXTRAS & 11.623 & 9.127 & & 186.291 & 6.595 & & \\
\hline \multirow{2}{*}{2012} & NORMAL & 102.448 & 90.920 & \multirow{2}{*}{216.830} & 537.240 & 4.472 & \multirow{2}{*}{726.106} & \multirow{2}{*}{942.936} \\
\hline & EXTRAS & 11.821 & 11.641 & & 183.828 & 566 & & \\
\hline \multirow{2}{*}{2013} & NORMAL & 105.080 & 101.384 & \multirow{2}{*}{229.961} & 417.560 & 5.976 & \multirow{2}{*}{588.629} & \multirow{2}{*}{818.590} \\
\hline & EXTRAS & 11.808 & 11.689 & & 163.621 & 1.472 & & \\
\hline \multirow{2}{*}{2014} & NORMAL & 117.984 & 114.592 & \multirow{2}{*}{253.350} & 579.224 & 8.272 & \multirow{2}{*}{716.819} & \multirow{2}{*}{970.169} \\
\hline & EXTRAS & 11.739 & 9.035 & & 128.217 & 1.106 & & \\
\hline
\end{tabular}

Fuente: elaboración propia sobre indicadores de nómina mensuales 2010-2014 - Gerencia de Talento Humano \&DO.

La tabla que antecede muestra las horas hombre de trabajo tanto del personal administrativo como operativo, en la que se evidencia las horas acumuladas anuales generadas en horas de trabajo normal y aquellas que se generaron como extras. De acuerdo al análisis promedio del periodo en estudio se tiene que el $76 \%$ de las horas hombre trabajadas corresponden al personal operativo y el $24 \%$ de horas hombre trabajadas son en actividades administrativas, de la cuales son horas normales generadas por los obreros el $58 \%$ y el $18 \%$ en horas extras, mientras que el $24 \%$ resulta de $21 \%$ horas normales y $3 \%$ de horas extras.

Figura 3. Composición de horas de trabajo (porcentaje) periodo 2010-2014

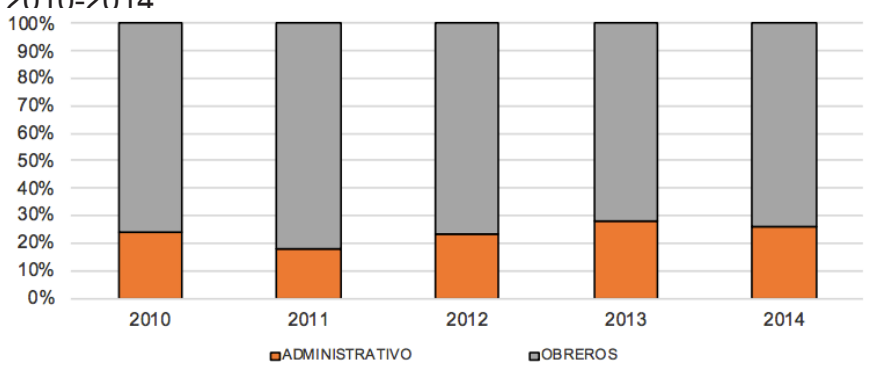

Fuente: elaboración propia.

La composición de horas de trabajo corresponde en un mayor porcentaje a las horas generadas por los obreros. Es importante resaltar que la planta industrial se mantiene operativa las 24 horas del día, los 365 días del año, para lo cual dispone de personal operativo distribuido en turnos rotativos. Como se puede apreciar en la figura 3, en el año 2010 el 24\% de las horas trabajadas corresponde al personal administrativo y el $76 \%$ a personal operativo; en el año 2011 el personal administrativo registró 18\% 
y el operativo el 82\%; durante el 2012 la composición de horas de trabajo fue $23 \%$ del personal administrativo y el 77\% del personal operativo; mientas que, en el 2013 el $28 \%$ registró el personal administrativo y el $72 \%$ el personal operativo. Finalmente el año 2014 indica que el $26 \%$ de las horas trabajadas corresponde al personal administrativo y el $73 \%$ a los obreros.

Figura 4. Horas de productividad por actividad antes y después de implementar una nueva línea productiva (horas anuales acumuladas).

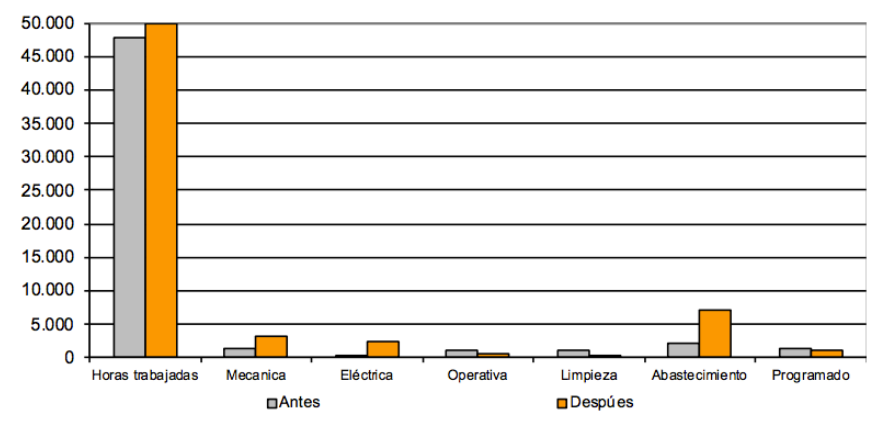

Fuente: elaboración propia sobre reporte de producción mensual 2010-2014.

Un aspecto importante de análisis en lo inherente a la productividad son las horas por actividad, con lo cual se pueden evaluar los cambios que generó la implementación de la nueva línea productiva, toda vez que elevó la capacidad instalada de la planta Chimborazo de la Unión Cementera Nacional UCEM CEM. En términos generales pasaron de 55.298 horas a 64.437 horas; es decir, un incremento general del 17\%.

Antes de la implementación del proyecto, 55.298 horas eran destinadas en el proceso productivo de operación de la trituradora primaria, trituradora secundaria, molino de crudo, horno, molino allis, molino miag1, molino miag2, pre trituración de clinker y ensacadora. De estas horas, 47.711 eran trabajadas efectivas, mientras que 1.360 horas se destinaban a trabajos de mantenimiento mecánico, 444 horas a mantenimiento eléctrico, 1.031 horas a temas operativos con el objetivo de que la capacidad instalada opere según estándares. Asimismo 1.167 horas se destinaban a la limpieza de áreas de trabajo, 2.121 horas corresponden a temas de abastecimiento de materias primas en las distintas maquinarias; por otras parte, 1.463 horas corresponden a mantenimiento programado, en virtud que las maquinarias habían superado su vida útil y requerían intervención para mantenimiento correctivo y preventivo.
Una vez implementada la nueva capacidad productiva, se registran 64.437 horas destinadas en el proceso productivo de la operación de la trituradora primaria, trituradora secundaria, molino de crudo, horno, pre trituración de clinker, molino de cemento CM4, molino de cemento CM3, molino de cemento CM2, molino de cemento CM1 y paletizadora. De la cuales 49.884 son horas trabajadas efectivas, mientras que 3.104 horas se destinaban a trabajos de mantenimiento mecánico, 2.477 horas a mantenimiento eléctrico, 535 horas se destinaban a temas operativos para que la capacidad instalada opere según estándares, 307 horas se destinaban a la limpieza de áreas de trabajo, 7.118 horas corresponden a temas de abastecimiento de materias primas en las distintas maquinarias; de otro lado, 1.012 horas corresponden a mantenimiento programado correctivo y preventivo para alagar la vida útil de las nuevas maquinarias.

Tabla 2. Análisis de la productividad según actividad antes y después de la implementación de la nueva línea de producción (horas - \%).

\begin{tabular}{c|c|c|c|c|}
\multirow{2}{*}{} & \multicolumn{2}{|c|}{ PROYECTO } & \multirow{2}{*}{ RESULTADO } \\
\cline { 2 - 3 } & SIN & CON & \\
\hline $\begin{array}{c}\text { HORAS } \\
\text { TRABAJADAS }\end{array}$ & 47.711 & 49.884 & & $5 \%$ \\
\hline MECANICA & 1.360 & 3.104 & & $128 \%$ \\
\hline ELECTRICA & 444 & 2.477 & & $457 \%$ \\
\hline OPERATIVA & 1.031 & 535 & $\wp$ & $-48 \%$ \\
\hline LIMPIEZA & 1.167 & 307 & $\wp$ & $-74 \%$ \\
\hline ABASTECIMIENTO & 2.121 & 7.118 & $\bullet$ & $236 \%$ \\
\hline PROGRAMADO & 1.463 & 1.012 & $\wp$ & $-31 \%$ \\
\hline
\end{tabular}

Fuente: elaboración propia sobre reporte de producción mensual 2010-2014

El resultado de las variaciones favorables para la producción en relación a los cambios experimentados por la nueva línea de producción, en lo referente a las horas de actividad destinadas, se tiene que las horas efectivas de trabajo incrementaron en un 5\%. También la nueva capacidad instalada permitió la reducción de un $48 \%$. En los temas operativos, la limpieza de las áreas de trabajo disminuyó en un $74 \%$, dado que los procesos son más automatizados y las nuevas máquinas poseen sistemas que merman el desperdicio de materias primas. Por ser maquinaria y equipos nuevos, las horas destinadas a mantenimiento programado también se contraen en un $31 \%$. Sin embargo, las horas destinadas a mantenimiento mecánico y eléctrico han incrementado en un $128 \%$ y $457 \%$ respectivamente, dado que para la 
operación de los nuevos equipos se requiere de personal técnico capacitado y, mientras adquirían experticia, era necesaria la intervención de personal de mantenimiento mecánico y eléctrico. Por otra parte, las horas destinadas al abastecimiento de materias primas han incrementado en un $236 \%$, lo que muestra la necesidad de trabajar en el incremento del stock de materias primas como clinker, puzolana, yeso y arcilla.

\section{Función de producción Cobb Douglas de UCEM CEM.}

Conforme la información primaria disponible de la Unión Cementera Nacional UCEM CEM - planta Chimborazo, se construyó la base de datos que se presenta a continuación, en función a los factores de capital y trabajo.

Tabla 3. Datos para estimación de la función de producción de la UCEM C.E.M. (Toneladas métricas - dólares). Periodo 20132014.

\begin{tabular}{cccc}
\hline PERIODO & $\begin{array}{c}\text { VOLUMEN de } \\
\text { PRODUCCIÓN (TM) }\end{array}$ & $\begin{array}{c}\text { FACTOR } \\
\text { CAPITAL (USD.) }\end{array}$ & $\begin{array}{c}\text { FACTOR } \\
\text { TRABAJO (USD.) }\end{array}$ \\
\hline Ene-13 & $26.097,69$ & $46.524 .721,16$ & $309.017,07$ \\
\hline Feb-13 & $22.348,28$ & $46.524 .721,16$ & $317.595,26$ \\
\hline Mar-13 & $25.254,40$ & $46.524 .721,16$ & $303.782,77$ \\
\hline Abr-13 & $26.065,00$ & $46.538 .877,48$ & $308.562,46$ \\
\hline May-13 & $38.679,00$ & $46.581 .397,49$ & $299.974,43$ \\
\hline Jun-13 & $28.012,00$ & $46.582 .814,88$ & $312.588,37$ \\
\hline Jul-13 & $32.726,00$ & $46.593 .448,49$ & $297.057,45$ \\
\hline Ago-13 & $37.435,00$ & $46.593 .448,49$ & $297.628,34$ \\
\hline Sep-13 & $40.958,86$ & $46.771 .566,02$ & $306.829,49$ \\
\hline Oct-13 & $44.261,54$ & $46.771 .566,02$ & $273.301,33$ \\
\hline Nov-13 & $41.574,23$ & $46.771 .566,02$ & $310.405,40$ \\
\hline Dic-13 & $43.156,38$ & $47.498 .576,31$ & $253.865,12$ \\
\hline Ene-14 & $35.588,70$ & $47.463 .435,55$ & $277.795,43$ \\
\hline Feb-14 & $38.674,47$ & $47.470 .353,61$ & $275.827,17$ \\
\hline Mar-14 & $40.581,80$ & $47.470 .353,61$ & $275.415,00$ \\
\hline Abr-14 & $43.806,89$ & $47.514 .506,31$ & $270.004,28$ \\
\hline May-14 & $47.727,63$ & $48.368 .672,87$ & $295.505,00$ \\
\hline Jun-14 & $42.837,64$ & $48.383 .693,30$ & $293.072,00$ \\
\hline Jul-14 & $46.487,20$ & $48.535 .818,56$ & $301.099,00$ \\
\hline Ago-14 & $47.127,20$ & $48.519 .350,38$ & $300.352,43$ \\
\hline Sep-14 & $52.620,00$ & $48.558 .773,00$ & $305.021,55$ \\
\hline Oct-14 & $50.174,00$ & $48.751 .710,16$ & $297.518,00$ \\
\hline Nov-14 & $48.896,88$ & $48.838 .397,97$ & $285.074,99$ \\
\hline
\end{tabular}

Dic-14 $50.619,54$ $48.924 .412,07$ $277.069,60$

Fuente: elaboración propia con datos de la Unión Cementera Nacional UCEM CEM. - planta Chimborazo, siendo el factor capital tomado de los estados financieros mensuales (activos fijos) y el factor trabajo hace referencia al costo de nómina.

Una vez estimado el modelo, se tiene que la función de producción de la Unión Cementera Nacional UCEM CEM - planta Chimborazo, en función a los factores de trabajo y capital, su volumen de producción está definido así:

$\ln Y i=\ln -139.2899+9.242564 \ln X 2 i-1.073556 \ln X 3 i+u i$

In Volumen de producción $=\ln -139.29+9.24 \ln$ factor trabajo $-1.07 \ln$ factor capital $+u i$

A partir de la regresión logarítmica de la función de producción de la Unión Cementera Nacional UCEM CEM - planta Chimborazo, es posible interpretar los coeficientes de la siguiente forma:

$\beta 1=-139.289$ indica que empresa se ve afectada si no se invierte en capital y trabajo, lo cual provocaría que la producción disminuya en -139.289 toneladas métricas.

$\beta 2=9.242$, se interpreta como la tasa de variación del volumen producido, es decir, que por cada 9.242 dólares que se inviertan en trabajo, la producción de cemento incrementará en una tonelada métrica.

$\beta 3=-1.073$, se interpreta como la tasa de variación del capital, es decir, que por cada reducción del el capital en - 1.073 dólares, la producción incrementará en una tonelada.

El p-valor del modelo y de las distintas variables indica la significatividad general del modelo. El coeficiente de determinación muestra que el modelo de producción de la Unión Cementera Nacional UCEM C.E.M planta Chimborazo, es explicado en un $66,51 \%$ por las variaciones de los factores o insumos trabajo y capital. $\mathrm{Al}$ respecto es importante recordar que en la producción también intervienen otros factores no fueron incluidos en el presente modelo. 


\section{Discusión y conclusiones}

El estudio pone de manifiesto la falta de utilización de la capacidad instala para la producción en Unión Cementera Nacional UCEM CEM - planta Chimborazo. Es posible que la planta explote el factor capital de manera más eficiente. La función de producción Cobb Douglas de la Unión Cementera Nacional UCEM CEM - planta Chimborazo, demuestra que el costo marginal de los factores trabajo y capital es 9.242 y -1.073 dólares respectivamente.

La situación económica de la planta Chimborazo de la Empresa Unión Cementera Nacional UCEM CEM, se encuentra en crecimiento y evolución debido a la ampliación de la capacidad productiva, lo cual le permite contar con un volumen de producción superior, a la vez que mejora su oferta en el mercado nacional e internacional. Analizados los factores de capital y trabajo, se establece que la empresa debe invertir en estos insumos constantemente para tener un incremento de la producción. Sin embargo, debe trabajar para no tener capital ocioso dado que, al no alcanzar el nivel máximo de producción, se desperdicia su capacidad instalada. Es por ello que el modelo indica que se requiere una reducción del capital.

\section{Referencias}

1. Aguado, J. C. (2011). Curso fundamental de microeconomía. Primera Edición, Delta Publicaciones, España.

2. Acevedo Fernández, E. (2009). PIB potencial y productividad total de los factores: Recesiones y expansiones en México. Economía mexicana. Nueva época, 18(2), 175-219. Disponible en: http://www.scielo.org.mx/scielo.php?script $=$ sci arttext\&pid=S1665-20452009000200002\&lng=es\&t $\operatorname{lng}=\mathrm{es}$.

3. Azofeifa, A. G. y Villanueva, M. (1996). Estimación de una función de producción: Caso de Costa Rica. DIE-PI-06-95/R, Departamento de Investigaciones Económicas. Banco Central de Costa Rica.

4. Bellod, J. F. (2011). La función de producción Cobb Douglas y la economía española. REC Revista de Economía Crítica, 12, 9-38. Disponible en: http:// www.revistaeconomiacritica.org/sites/default/files/
revistas/n12/REC12_Articulo_2_bellod.pdf

5. Camacho Murillo, A. y Ríos Hernández, H. (2011). Competencia intra-industria, aglomeraciones regionales y crecimiento económico: caso industria de alimentos. Revista de la Facultad de Ciencias Económicas: Investigación y Reflexión, XIX (1), 5571. Disponible en: https://www.redalyc.org/articulo. oa? id=90922732004

6. Céspedes, N., Aquije, M. E., Sanchez, A., y VeraTudela, R. (2014). Productividad sectorial en el Perú: Un análisis a nivel de firmas. Revista Estudios Económicos, 28, 9-26. Disponible en: http://www. bcrp.gob.pe/docs/Publicaciones/Revista-EstudiosEconomicos/28/ree-28-cespedes-aquije-sanchezveratudela.pdf

7. Cortázar, A. y Montaño, E. (2011). La función Cobb Douglas en la producción de algodón del Valle de Juárez: Aplicación a factores definidos e interpretación específica de resultados. Revista Fuente, 9, 137-150. Disponible en: http://fuente.uan. edu.mx/publicaciones/03-09/9.pdf

8. Dominick, S. (2009). Microeconomía. Cuarta edición, McGraw-Hill, México.

9. Méndez, J. A., Méndez, J. M., y Hernández H. A. (2013). Productividad total de los factores, cambio técnico, eficiencia técnica y PIB potencial en latinoamérica. Semestre económico, 16(34), 65-91. Disponible en: http://www.scielo.org.co/pdf/seec/ v16n34/v16n34a4.pdf

10. Nicholson, W. (2008). Teoría microeconómica: Principios básicos y ampliaciones. Novena Edición, Cengage Learning Editores, México.

11. Parra Osorio, J. F. (2012). Una función de producción para el teatro en Colombia. Cuadernos de Economía, 31(56), 149-177. Disponible en: http://www.scielo. org.co/scielo.php?script=sci_arttext\&pid=S0121$47722012000100006 \& \operatorname{lng}=$ en $\&$ tlng=es. 